\title{
Zu Begriff und Verfahren des Kommentars bei Nietzsche und Adorno
}

Christen, Felix

DOI: https://doi.org/10.1515/9783110304053-007

Posted at the Zurich Open Repository and Archive, University of Zurich

ZORA URL: https://doi.org/10.5167/uzh-144116

Book Section

Published Version

Originally published at:

Christen, Felix (2017). Zu Begriff und Verfahren des Kommentars bei Nietzsche und Adorno. In: Endres, Martin; Pichler, Axel; Zittel, Claus. Text/Kritik: Nietzsche und Adorno. Berlin, Boston: De Gruyter, 273-297.

DOI: https://doi.org/10.1515/9783110304053-007 


\section{Felix Christen}

\section{Zu Begriff und Verfahren des Kommentars bei Nietzsche und Adorno}

Obgleich Adorno die Philologie mitunter mit einer Rekonstruktion der intentio auctoris synonym setzt und dementsprechend verwirft (vgl. etwa ME, GS 5, S. 343; ÄT, GS 7, S. 226 und S. 507), beziehen sich seine Kritik an der Sprachvergessenheit der Philosophie ebenso wie sein Denken der Literatur unter anderem auf den auch philologisch zu verstehenden Begriff des Kommentars. Die Sprachlichkeit der Philosophie ist dabei auch und gerade dort, wo sie implizit bleibt, Voraussetzung textueller Verfahren der Kritik, die diese Sprachlichkeit in den Blick rücken. Gesteht die Philosophie "[d]urch die sei's offenbare, sei's latente Gebundenheit an Texte« ein, "was sie unterm Ideal der Methode vergebens ableugnet, ihr sprachliches Wesen« (ND, GS 6, S. 65), so knüpft daran eine Kritik an, die das sprachliche Wesen expliziert. Wenn Adorno in der Einleitung der Negativen Dialektik deshalb die Rhetorik als Urverdängtes der Philosophie rehabilitieren will in dem ebenso allgemeinen wie präzisen Sinn eines »durch nichts ganz zu zerbrechenden Zusammenhang[s] « (ND, GS 6, S. 66) von Denken und Sprache, ${ }^{1}$ findet sich nicht nur im Prinzip der Deutung, wie es in der Frankfurter Antrittsvorlesung (vgl. PhF, GS 1, S. 334-344) und in der Negativen Dialektik (vgl. ND, GS 6, S. 64) exponiert ist, sondern auch in Adornos Begriff des Kommentars ein Verfahren der "Methexis der Philosophie an der Tradition" (ND, GS 6, S. 64). Dieses Kommentarverhältnis, das Adorno zur philosophischen Überlieferung entwirft, gilt mit den notwendigen Änderungen auch für seine Auseinandersetzungen mit der Literatur, die trotz des programmatischen einleitenden Versuchs über den Essay nicht als ১Essays«, sondern als »Noten« tituliert sind - was sich, den kommentierenden Noten in Goethes Noten und Abhandlungen zu besserem Verständniß des West-östlichen Divans nicht unähnlich (vgl. Goethe 1994, S.139), durchaus auch im Sinne eines Kommentars verstehen

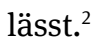

Die philosophische Wendung des philologischen Kommentarbegriffs bei Adorno bezieht sich explizit, obschon nicht unkritisch, auf Walter Benjamin (vgl. KG, GS 10, S. 244); ein in verwandter, wenn auch nicht identischer Weise philosophisch gewendeter Kommentarbegriff findet sich aber bereits bei Nietz-

1 Vgl. zur Aufwertung der Rhetorik bei Adorno auch Scholze 2000, S. 289-294.

2 Zum Goethe-Bezug siehe NzL, GS 11, S. 329. Eine Aufgliederung möglicher Bedeutungen des Titels »Noten zur Literatur« findet sich bei Plass 2007, S. 185f., Anm. 38. 
sche. Der frühe Nietzsche, der in seiner programmatischen Baseler Antrittsvorlesung die Philologie an die Stelle der Philosophie treten sieht (vgl. KGW II/1, S. 268), verwendet den philologischen Kommentarbegriff in privater Korrespondenz ebenso wie in der schriftlichen Selbstdarstellung anlässlich des Rufs an die Universität Basel (vgl. KGW I/5, S. 55). In den 1880er Jahren steht der Kommentarbegriff dann nicht nur prominent - und in der Forschung am stärksten beachtet - in der Vorrede der drei Abhandlungen Zur Genealogie der Moral, deren dritte als »Commentar « zum vorangestellten Aphorismus bestimmt wird (GM Vorrede 8, KSA 5, S. 256); Nietzsche glaubt in brieflichen Äußerungen auch, das Verhältnis seiner Zarathustra-Dichtung zu seinen übrigen Schriften als Verhältnis von Text und Kommentar begreifen zu können (vgl. Groddeck 1983, S. 24). Die Grenze einer säkular-philologischen zur theologischen Kommentartradition überschreitend, ${ }^{3}$ setzt er dabei den Zarathustra als einen gleichsam heiligen Text an, als »ein neues sheiliges Buch«" (KGB III/1, Bf. 404), das zu kommentieren ist. Dieses emphatische Kommentarverhältnis zum Text, das »[p]rofane Texte wie heilige« anschaut (NzL, GS 11, S. 129), gilt, obschon freilich nicht aufs eigene Werk bezogen, auch für Adornos alexandrinische Philosophie, »wo der Gedanke das unwiederbringliche Urbild heiliger Texte säkularisiert« (ND, GS 6, S. 64).

Im Folgenden werden mit Blick auf diese philosophische Wendung Begriff und Verfahren des Kommentars zunächst bei Nietzsche (Teil I), dann bei Adorno (Teil II) untersucht. Dabei soll für die Kommentarpraxis, die mit dem Begriff eng zusammenhängt, auch gefragt werden, ob sie sich nicht nur auf die explizit als Kommentar gekennzeichneten oder kommentierend verfahrenden Texte Nietzsches und Adornos erstreckt, also ein Textverfahren ist, sondern sich auch als Schreibverfahren verstehen lässt (Teil III). Ist die Herausbildung einer gedanklichen Logik der Kommentierung eng mit der Räumlichkeit der Schrift verbunden, ${ }^{4}$ lassen sich bei Nietzsche und Adorno nicht nur in publizierten Texten, die als Kommentar angelegt sind, sondern auch in der Topographie der Manuskripte kommentierende Relationen finden. Gefragt wird also zum einen nach dem Begriff des Kommentars bei beiden Autoren, zum anderen nach der Kommentaranlage publizierter Texte ebenso wie nach kommentierenden Elementen in der Struktur von Entwürfen und Notaten.

$3 \mathrm{Zu}$ Entwicklung und Struktur der theologischen Kommentartradition siehe z. B. die entsprechenden Beiträge bei Kästle/Jansen 2014.

4 Vgl. Schironi 2012 sowie die grundlegende Darstellung der alexandrinischen Philologie bei Pfeiffer 1968, insbes. das Kapitel zu Aristarch, S. 210-233. 
Wenige Wochen nachdem er aus gesundheitlichen Gründen ein Gesuch um Entlassung von seiner altphilologischen Professur eingereicht hat, schreibt Nietzsche an Overbeck: "Seit meiner letzten Karte die meiste Zeit zu Bett gelegen: dies ein Commentar, zu dem ich mir den Text ersparen darf.« (KGB II/5, S. 420) ${ }^{5}$ Den Text zu diesem Kommentar hat Nietzsche bereits in seinem Entlassungsgesuch verfasst. Während im offiziellen Schreiben der Gesundheitszustand Gegenstand ausführlicher Erörterungen ist (vgl. KGB II/5, S. 411f.), belässt es Nietzsche in der Intimität der privaten Korrespondenz bei einem lakonischen Kommentar ohne Text. Der Gedanke, einen Kommentar zu verfassen, zu dem ein Text nicht unmittelbar vorliegt, ist bei Nietzsche allerdings nicht nur im privaten Austausch zu finden. Mehrere Werke aus Nietzsches >Dekalog ‘ - den zehn Schriften, die er selbst in Ecce homo als sein Gesamtwerk kanonisieren will (vgl. Stingelin 2002, S. 80f.; Sanchiño Martínez 2013, S. 285-287) -, gruppieren sich in seinen Augen, wie er mehrfach brieflich erläutert, wie Kommentare um den Zarathustra als den Text schlechthin, wobei einige der Kommentare der Entstehung des Textes vorausgehen. Kurz nach der Niederschrift des ersten Teils von Also sprach Zarathustra schreibt Nietzsche an Köselitz:

Es ist ein curiosum: ich habe den Commentar früher geschrieben als den Text. Versprochen ist Alles schon in »Schop<enhauer > als Erz<ieher «; es war aber ein gutes Stück Weg von »Menschl<iches〉, Allzum<enschliches»« bis zum »Übermenschen« zu machen. Wenn Sie jetzt einen Augenblick an die »fröhl<iche> Wiss<enschaft «« zurückdenken wollen, so werden Sie lachen, mit welcher Sicherheit, ja impudentia darin die bevorstehende Geburt »annoncirt« wird. - (KGB III/1, Bf. 405)

Wenn Nietzsche feststellt, dass er »den Commentar früher geschrieben« habe "als den Text«, so überschreitet er damit eine der scheinbar selbstevidenten Vorgaben eines philologischen Kommentars, wie er sich in der alexandrinischen Schule seit dem 3. Jahrhundert v. Chr. ausgebildet hat. Nicht mehr die temporale Struktur der Nachträglichkeit zeichnet den Kommentar als Supplement zum Text aus - also eine "Schließung« des zu kommentierenden Textes

5 Die folgenden Ausführungen zu Nietzsche gehen auf einen Vortrag zurück, den ich im Rahmen eines von mir geleiteten Workshops u. d. T. »Interpretation « und `Commentar«. Zwei philologische Begriffe in Nietzsches Philosophie« im Oktober 2015 am Department of Germanic Languages and Literatures der Harvard University gehalten habe.

6 Vgl. auch KGB III/1, Bf 504. Siehe dazu Stingelin 2002, S. 100, der in der Vorgängigkeit des Kommentars ein »Umkehrverhältnis« sieht, »mit dem der Autor dem Leser zuvorkommen will«. 
und der »Späthorizont eines posthistoire« im Kommentar, wie Assmann es genannt hat (J. Assmann 1995, S. 28f.) -, sondern eine Differenzierung der Texte, die den einen Text kanonisiert, indem er durch andere Texte sich des Kommentars als würdig erweist. Nicht die Kanonisierung ist Bedingung des Kommentars, sondern der Kommentar Bedingung der mit diesem beabsichtigten Kanonisierung. ${ }^{7}$ Nietzsche kann den Zarathustra als klassischen Text bestimmen, weil dieser der ausführlichen Kommentare bedarf, ja diese Kommentare z. T. bereits vorliegen. ${ }^{8}$ Allerdings ist die Autorschaft von Text und Kommentar bei Nietzsche identisch; er selbst bestimmt, was Text ist, was Kommentar, die beide Teile seiner Schriften sind und also gewissermaßen ein Selbstverhältnis des Autors Nietzsche anzeigen.

Während Nietzsches Begriff der Interpretation große Aufmerksamkeit zuteilgeworden ist (vgl. u. a. Figl 1982, Schrift 1990, Abel 1998, Figal 2000), wird sein Begriff des Kommentars eher am Rande, wenn überhaupt, vermerkt oder mit Skepsis in Hinblick auf seine Tauglichkeit für eine Werkanalyse beurteilt (vgl. Sommer 2013a) und ausführlicher nur in Zusammenhang mit der dritten Abhandlung zur Genealogie der Moral zur Kenntnis genommen (zuletzt von Westerdale 2013, S. 99-122). Demgegenüber ist es Christian Bennes großes Verdienst, den wissenschaftshistorischen Kontext der Herausbildung und Entwicklung von Nietzsches Denken in der Philologie erkannt und nicht zuletzt unter Verweis auf den philologischen Begriff des Kommentars das Lesen als Gegenbegriff zum Interpretieren erläutert zu haben (vgl. Benne 2005, S. 197-212). Gerade der Begriff des Kommentars ist bei Nietzsche philologisch geprägt; der Altphilologe Nietzsche kennt die spezifisch philologische Verwendungsweise des Kommentarbegriffs und greift auf sie zurück. Zugleich erweitert er diesen im engeren Sinne philologischen Begriff und entwirft damit eine Möglichkeit des Umgangs mit Texten, die über den Stellenkommentar hinausgeht in Richtung auf ein genuin philosophisches Unternehmen, das allerdings einen philologischen Kern bewahrt.

Die Kommentierung schwieriger Passagen klassischer Werke erlernt Nietzsche bereits als Schüler der altsprachlich ausgerichteten Landesschule Pforta,

7 Es ist diese Struktur, die Foucault in seiner Antrittsvorlesung am Collège de France als Funktion des Kommentars bestimmt - als »Prinzip der Abstufung«, wie es bei Foucault heißt (Foucault 1991, S. 18.)

8 Im Zarathustra selbst ähneln dabei Zarathustras Lehrversuche mitunter der »Form der mündlichen, unterweisenden Textauslegung « (etwa in der Erläuterung der Erzählung vom »Biss der Natter«; Za I, KSA 4, S. 87-89), die Assmann mit einem Begriff aus der Apostelgeschichte »Hodegetik« nennt, also Lehrdisputation, bei der der Lehrer dem Schüler den Kommentar zum Text abgibt (J. Assmann 1995, S. 30f.). 
wo »mitunter spezifisch philologische Aufgaben gestellt« werden, »zB. kritische Commentare über bestimmte sophokleische oder äschylische Chorgesänge. Dann ist es ein besondrer Vorzug der Schulpforte, daß unter den Schülern selbst eine angestrengte und mannichfache Lektüre griechischer und römischer Schriftsteller zum guten Ton gehört.« (KGW I/5, S. 55f.) Das Prinzip des kritischen Kommentars, verbunden mit der intensiven und wiederholten Lektüre, wird von Nietzsche als eine philologische Praxis verstanden, die zugleich weitreichende Implikationen hat. Denn gerade im Modell von Text und Kommentar findet der Philologie Nietzsche die Grundstruktur des klassischen Werks, das der späte Nietzsche sich in Selbstkommentierungen erschreiben will. In einer der nachgelassenen »Notizen / zu / Wir Philologen« von 1875 heißt es:

Mir steht nun einmal fest, dass eine einzige Zeile geschrieben zu haben, welche es verdient, von Gelehrten späterer Zeit commentirt zu werden, das Verdienst des grössten Critikers aufwiegt. Es liegt eine tiefe Bescheidenheit im Philologen. Texte verbessern ist eine unterhaltende Arbeit für Gelehrte, es ist ein Rebusrathen; aber man sollte es für keine zu wichtige Sache ansehen. (NL 1875, 3[31], KSA 8, S. 23)

In dieser Notiz zeichnet sich der Übergang ab von einem auch nach Veröffentlichung der Geburt der Tragödie noch immer als >Gelehrter〈bzw. >Critiker` tätigen Nietzsche zu einem Autor, der es mit zumindest seiner einzigen Zeile`, wie es heißt, »verdient, von Gelehrten späterer Zeit commentirt zu werden«. Die kommentierenden Philologen werden damit zwar auf den zweiten Platz verwiesen, und Emendation und Konjektur (»Texte verbessern«) sind »keine $\mathrm{zu}$ wichtige Sache« - eine Zeile, die es verdient hat, kommentiert zu werden, wiegt an Bedeutung auch den bedeutendsten Kommentar auf. Aber ebendieser Kommentar zeigt umgekehrt ihre Bedeutung an. Nietzsche schüttelt als philosophischer Autor den Kommentar denn auch keineswegs als nur sekundäres Phänomen ab; vielmehr will er ihn in sein Werk integrieren. Dabei umreißt er als Voraussetzung, um den »Text «, nämlich den Zarathustra, überhaupt lesen zu können, das jahrelange Studium der ১Kommentare`, also seiner anderen Schriften: »In der That habe ich das Kunststück (und die Thorheit) ıbegangen`, die Commentare eher zu schreiben als den Text. - Aber wer hat sie denn gelesen? Ich meine: jahrelang studirt? Ein Einziger, so viel ich weiß: dafür hat er nun auch seine Freude am Texte. «(KGB III/1, Bf. 404). ${ }^{9}$

Dass der Kommentar mitunter vor dem Text entsteht, gilt nicht nur für Nietzsches Werk, wie es ihm selbst in seinen brieflichen Äußerungen erscheint.

9 Im selben Brief an Malwida von Meysenbug verweist Nietzsche auf den Zarathustra als »neues > heiliges Buch« (KGB III/1, Bf. 404). 
Ebenso ist in der dritten Abhandlung zur Genealogie der Moral der erste Abschnitt, dessen »Commentar" die Abhandlung laut Nietzsches Vorrede ist (GM Vorrede 8, KSA 5, S. 256), erst nachträglich hinzugefügt worden (vgl. KSA 14, S. 380). Trotz umgekehrter Reihenfolge der Entstehung kehrt Nietzsche, wie bei einem Kommentar zu erwarten, immer wieder zum zu kommentierenden Text zurück - oder legt, bedenkt man die Textentstehung mit, den ersten Abschnitt so an, dass er als Text zum nachfolgenden Kommentar erscheint. Obschon die dritte Abhandlung einen fortlaufenden Gedankengang entwickelt - was, wie Stegmaier (2012, S. 72) feststellt, üblicherweise gerade nicht Kennzeichen eines Kommentars ist -, entsprechen die im ersten Abschnitt exponierten verschiedenen Bereiche der Frage, was asketische Ideale bedeuten, bis in den Wortlaut hinein den folgenden Ausführungen (vgl. Benne 2005, S. 205, sowie Westerdale 2013, S. 107f.; Nachweise im Einzelnen erbringt Wilcox 1997, S. 605-607). Die Erläuterung zur Kommentarstruktur der dritten Abhandlung, die Nietzsche in der Vorrede zur Genealogie vorbringt, korrespondiert dabei mit der Bestimmung des philologischen Kommentars, der neben seiner im engeren Sinn textkritischen Funktion üblicherweise dort benötigt wird, wo sich eine überlieferte Textstelle dem Verstehen entzieht, wo sie dunkel erscheint (vgl. Fuhrmann 1985, S. 42-47). Denn Nietzsche weist in der Vorrede auf die Probleme bei der Auslegung des Aphorismus hin, die zugleich durch den Kommentar ausgeräumt werden sollen: »Wenn diese Schrift irgend Jemandem unverständlich ist und schlecht zu Ohren geht, so liegt die Schuld, wie mich dünkt, nicht nothwendig an mir« (GM Vorrede 8, KSA 5, S. 255) - Letzteres gerade deshalb nicht, weil Nietzsche mit seinem »Commentar« ein »Muster von dem dargeboten« hat, was er »Kunst der Auslegung « nennt, und er damit der »Schwierigkeit« der »aphoristische[n] Form « (GM Vorrede 8, KSA 5, S. 255) begegnet. ${ }^{10}$

Setzt man die Mustergültigkeit dieses Kommentars voraus, so ist auch Nietzsches Prinzip eines vornehmlich zu kommentierenden, gleichsam heiligen Textes, des Zarathustra, in ihm enthalten. Will man einen Kommentar nicht zuletzt als direkt sich auf den Wortlaut beziehende Relation zwischen zwei Tex-

10 Ein `Muster ist ein Probestück, das nachgeahmt werden soll, und zwar, wie es im Grammatisch-kritischen Wörterbuch von Adelung heißt, »besonders so fern« es »zugleich die Art und Weise der Nachahmung zeiget«, also die Weise der Verfertigung selbst mit darstellt (Adelung 1793-1801, Bd. 3, Sp. 332). Deshalb ist es auch ein Fachwort der Textilsprache, das die Verfertigung einer bestimmten Stoffstruktur oder -gestalt vorgibt, aber im Verhältnis zu dem anzufertigenden Stück kleiner ist. Das »Muster«, das Nietzsche vorlegt, hat, so gesehen, Vorbildcharakter - es führt im Kleinen vor, was Nietzsche als Struktur der Auslegung überhaupt umreißen will. Ebendieses Verhältnis der Auslegung, für das man sich am »Muster« orientieren kann, bezeichnet Nietzsche als »Commentar«. 
ten bestimmen, ist davon auszugehen, dass der kommentierte Aphorismus, wie erläutert, der erste Abschnitt der dritten Abhandlung zur Genealogie der Moral ist - und nicht, wie zuweilen vertreten, das der dritten Abhandlung noch vor dem ersten Abschnitt auf einer separaten Seite vorangehende Zarathustra-Zitat, das zunächst dem ganzen Buch hätte vorangestellt werden sollen. ${ }^{11}$ Gleichwohl entsteht durch das Zarathustra-Zitat eine Art Abstufung des Kommentars, in der die erste Stelle dem Zarathustra gebührt. Zum Zarathustra-Zitat steht der folgende Abschnitt 1 der dritten Abhandlung, der von Nietzsche immer wieder betonten Unzugänglichkeit seines Lehrgedichts entsprechend, in einem nur schwer nachvollziehbaren Verhältnis, während dieser erste Abschnitt auf wesentlich leichter nachzuvollziehende Weise in der gesamten folgenden Abhandlung kommentiert wird. - Dazu verhält sich wiederum die Vorrede als Kommentar nicht nur inhaltlicher, sondern struktureller Art, indem sie das Kommentarverhältnis selbst kommentiert. Dass es dabei keineswegs eine Überstrapazierung von Nietzsches Kommentarbegriff ist, die Relation des Kommentars der dritten Abhandlung zur Vorrede wiederum als Kommentarverhältnis zu begreifen, ist nicht nur strukturell begründbar, sondern wird von Nietzsche in einem Brief an Meta von Salis vom September 1887 - während des Drucks der dritten Abhandlung der Genealogie - explizit so erläutert. Nietzsche bezeichnet die späte Vorrede zur Geburt der Tragödie und die Vorrede zur Genealogie der Moral als >Commentieren (KGB III/5, Bf. 908).

Wenn Nietzsche vorschwebt, dass sich seine Schriften wie ein Kommentar und wie Kommentarschichten, insofern in den Schriften selbst wiederum kommentierende Relationen zu vermerken sind - um sein Hauptwerk Also sprach Zarathustra legen, ${ }^{12}$ so gilt dies auch für die unmittelbar vor der Genealogie der Moral abgeschlossene Schrift Jenseits von Gut und Böse: »Es [JGB] ist eine Art von Commentar zu meinem `Zarathustra`. Aber wie gut müßte man mich verstehn, um zu verstehn, in wie fern es zu ihm ein Commentar ist!« (KGB III/3, Bf. 768) Nietzsche unterstreicht im Brief "wie fern" - die Frage, die sich stellt, ist also gerade, was das Kommentarverhältnis bei Nietzsche bedeutet, ja $o b$ es

11 Vgl. die grundlegenden Argumente dazu bei Wilcox 1997 und Wilcox 1998 sowie die weiterführenden Überlegungen bei Benne 2005, S. 203-205, und bei Westerdale 2013, S. 104-107. In Notizheft N VII 3 (KGW IX/3, S. 32 und S. 34) finden sich Umschlagentwürfe zur Genealogie, die das Zarathustra-Zitat unmittelbar unter Buchtitel und Autornamen setzen.

12 Ein besonders komplexer Fall ist dabei der Dithyrambus »Unter Töchtern der Wüste«, der im Zarathustra bereits enthalten ist, dessen Vers "Die Wüste wächst: weh Dem, der Wüsten birgt! « (Za IV, KSA 4, S. 380) aber in der Version der Dionysos-Dithyramben anders als im Zarathustra als eigener Abschnitt nummeriert und exponiert wird: als ein gleichsam heiliger Text, dessen Kommentar die folgenden Strophen sind, wie Groddeck (1991, S. 58) ausführt. 
sich um ein Kommentarverhältnis handelt. ${ }^{13}$ Wie an dieser Briefstelle deutlich wird, ist der Kommentar für Nietzsche nicht nur eine Angelegenheit philologischer Methodik, sondern ein philologisch-philosophisches Problem. Das Verständnis von Nietzsches Kommentarbegriff hängt mit Fragen seines Denkens aufs Engste zusammen. Dabei lässt sich keineswegs leichthin bestimmen, worin denn denn nun das Kommentarverhältnis zum Zarathustra, das Nietzsche benennt, besteht (deshalb die Skepsis gegenüber Nietzsches Verwendung des Begriffs bei Sommer 2013a). Die Crux von Nietzsches Kommentarbegriff liegt gerade darin, dass er ein enges Verhältnis zwischen seinen Schriften postuliert, das zu erfassen seinerseits wiederum nach einer idealiter umfassenden Auseinandersetzung mit diesen Schriften, nach einem jahrelangen Studium, verlangt. Die Form des Kommentars ist dabei eine Setzung, die es grundsätzlich auch erlaubt, Neues und anderes zu sagen als der kommentierte Text unter der Voraussetzung, dass es eigentlich bereits im Text gesagt sei. ${ }^{14}$ Die Erläuterung der Kommentarstruktur, wie Nietzsche sie voraussetzt, erfordert deshalb nicht einfach eine Prüfung bestimmter Kriterien der Kommentarform, sondern verlangt nach deren Reflexion und damit nach jener philosophischen Wendung der Philologie, die bereits der frühe Nietzsche ins Auge fasst. In der Umkehrung von Senecas kritischem Diktum aus den Briefen an Lucilius, dass »das, was Philosophie war, zur Philologie geworden« sei (Seneca 1987, S. 651 [epist. 108, 23]), die Nietzsche in seiner Antrittsvorlesung »Homer und die klassische Philologie« vorträgt, ist nicht nur, wie Nietzsche selbst erläutert, ausgesprochen, »dass alle und jede philologische Thätigkeit umschlossen und eingehegt sein soll von einer philosophischen Weltanschauung « (KGW II/1, S. 268). Vielmehr deutet Nietzsches Sentenz "philosophia facta est quae philologia fuit» (KGW II/1, S. 268) auf ein Philosophisch-Werden der Philologie selbst. Dies gilt zumal für den Kommentar, der neben der Interpretation eine der beiden »besonders zentralen und ehrwürdigen philologischen Praktiken« ist (Gumbrecht 2003, S. 70), wobei der späte Nietzsche die Philologie gerade als Innehalten in der Deutung bestimmt, als "Ephexis in der Interpretation« (AC 52, KSA 6, S. 233; vgl. Groddeck 1991, S. XX; Fietz 1992, S. 398-418; Benne 2005, S. 198f.; Sommer 2013b, S. 245f.), aber keineswegs als Innehalten im Kommentar. Wo Nietzsche selbst eine Kommentierung unternimmt oder den Begriff des Kommentars mit Empha-

13 In der Aufzeichnung NL 1886/87, 6[4], KSA 12, S. 234, verwirft Nietzsche expressis verbis den Begriff des Kommentars zur Kennzeichnung von Jenseits von Gut und Böse und bezeichnet es stattdessen als »Glossarium « (siehe dazu Sommer 2013a).

14 Vgl. zu dieser Grundstruktur des Kommentars in rechtshistorischer und systematischer Hinsicht Vismann 2006, S. 349f. 
se verwendet, ist das Kommentieren gerade nicht von der Problematisierung betroffen, die einem willkürlich interpretierenden Zugriff gilt, der nicht zwischen Interpretation und Text $\mathrm{zu}$ unterscheiden weiß bzw. Interpretation und Text verwechselt (»das ist Interpretation, nicht Text«, lautet Nietzsches entsprechender Vorwurf; JGB 22, KSA 5, S. 37). Der Kommentar ersetzt den Text nichtim Gegensatz zur Interpretation, unter der, wenn sie »schlechte[ ] >Philologie«" ist, der Text verschwindet (JGB 22, KSA 5, S. 37; vgl. JGB 38, KSA 5, S. 56). ${ }^{15}$

Dabei ist es nicht zuletzt das Prinzip einer ausführlichen oder vollständigen Zitierung des zu kommentierenden Textes, das davor bewahrt, dass der Text unter dem Kommentar verschwände. Den vierten Abschnitt des Vorworts zu Ecce homo - einem Text, der expliziter als die Werke, die Nietzsche brieflich als Kommentare zum Zarathustra bestimmt, das Signum eines Selbstkommentars $\mathrm{zu}$ Nietzsches Schriften trägt ${ }^{16}$ - beginnt Nietzsche mit der emphatischen Setzung der Zarathustra-Dichtung als Text schlechthin, als ১höchstes` und ১tiefstes` Buch der Menschheit. Der erste Absatz endet mit einem kürzeren Zitat aus dem Zarathustra, auf das ein längeres folgt, worauf nach einigen Bemerkungen zum Ton des Zarathustra ein langes Zitat die Einleitung abschließt (vgl. EH Vorwort 4, KSA 6, S. 259-261). Auch der Abschnitt zum Zarathustra zitiert ausführlich, etwa das »Nachtlied« in voller Länge (vgl. EH Zarathustra 7, KSA 6, S. 345-347). Nietzsches Form der Selbstkommentierung ist hier nicht »anstatt«, sondern »an der Stelle « des zu kommentierenden Textes zu lesen. ${ }^{17}$ Sie tritt wie ein Interlinear- oder Marginalkommentar dem gleichsam heiligen Text, den sie allererst als solchen kanonisieren will, zur Seite.

15 In den genannten zwei Abschnitten von Jenseits von Gut und Böse wird die philologische Begrifflichkeit auf einen außerphilologischen Zusammenhang übertragen (die Natur und die Französische Revolution), wo sich andere Probleme als bei der Auslegung eines Textes - einer Handschrift, eines Drucktextes - stellen, wie Benne (2005, S. 208-212) betont. Mir geht es hier allerdings ausschließlich um das Problem der Textauslegung im engeren Sinn, für das die beiden Abschnitte in Jenseits von Gut und Böse nur durch ihre Erläuterung sschlechter Philologie`, die es freilich auch im Verhältnis zu überlieferten Texten gibt, nicht durch ihre Kritik an Physik und Geschichtswissenschaft von Interesse sind.

16 Vgl. zur Tradition des Selbstkommentars Aust 1993, A. Assmann 1995 sowie Geulen 2015.

17 So Goethes Terminologie bei der Bestimmung der höchsten Art der Übersetzung in den Noten und Abhandlungen zu besserem Verständniß des West-östlichen Divans (Goethe 1994, S. 281), die, als Interlinearversion verstanden, mit der Form des Interlinearkommentars korrespondiert (zur Nähe von Kommentar und Übersetzung siehe auch Vismann 2006, S. 350). 


\section{2}

In der Charakteristik Walter Benjamins bestimmt Adorno Benjamins Philosophie als »Kommentar und Kritik« und erkennt im kommentierenden Verhältnis zur Überlieferung ein wesentliches Element der Philosophie, das, in jüngster Zeit verschüttet, von Benjamin neu entdeckt und ins Zentrum eines Denkens gestellt wird, das ein religiöses Verhältnis zum Text in ein aufgeklärtes überträgt und gleichwohl auf struktureller Ebene bewahrt:

Auf jeden Fall hat er [Benjamin] an der Kabbala seinen Begriff des heiligen Textes orientiert. Philosophie bestand ihm wesentlich aus Kommentar und Kritik, und der Sprache, als der Kristallisation des »Namens«, schrieb er höheres Recht zu als das des Bedeutungsund selbst Ausdrucksträgers. Die Beziehung von Philosophie auf je kodifiziert vorliegende Lehrmeinungen ist ihrer großen Tradition weniger fremd, als Benjamin glauben mochte. Zentrale Schriften oder Partien von Aristoteles und Leibniz, von Kant und Hegel sind »Kritiken« nicht nur implizit, als Arbeit an aufgeworfenen Problemen, sondern als spezifische Auseinandersetzungen. Erst als die zur Branche zusammengeschlossenen Philosophen des eigenen Denkens sich entwöhnten, glaubte ein jeder dadurch sich decken zu müssen, daß er vor der Erschaffung der Welt anfing oder womöglich diese in die eigene Regie nahm. Demgegenüber hat Benjamin den entschlossenen Alexandrinismus vertreten und damit alle wurzelwütigen Affekte gegen sich aufgebracht. Die Idee des heiligen Textes transponierte er in eine Aufklärung, in die umzuschlagen nach Scholems Aufweis die jüdische Mystik selber sich anschickte. Sein Essayismus ist die Behandlung profaner Texte, als wären es heilige. (KG, GS 10, S. 244f.)

Während Adorno Benjamins Kommentardenken von Scholems Arbeiten zur Kabbala herleitet, ${ }^{18}$ umreißt er »Kommentar und Kritik» als »spezifische Auseinandersetzungen « mit überlieferten Texten, wie sie sich in der Philosophie seit der Antike finden. Die Kommentierung als philosophisches Verfahren wird denn auch bereits in Hegels Vorlesungen über die Geschichte der Philosophie im Teil zur »alexandrinischen Philosophie« historisiert (vgl. Hegel 2016, S. 127-134, S. 377-387; dazu Plass 2007, S. 33f.). Während der im Benjamin-Essay postulierte Kommentarbegriff sich durch das zentrale Prinzip der »Behandlung profaner Texte, als wären es heilige«, dem theologischen Kommentar annähert ebenso wie einem im engeren Sinne textkritischen Kommentar, der die Überlieferung absolut unverändert erhalten will, verwendet Adorno den Kommentar, den er in der Ästhetischen Theorie als Verfahren auch der eigenen Philosophie reklamiert (vgl. ÄT, GS 7, S. 289f.), durchaus auch im engeren Sinn eines Stellenkommen-

$18 \mathrm{Zu}$ den verschiedenen Aspekten von Benjamins Kommentarbegriff siehe Primavesi 1998, Vismann 2006 und Auerochs 2014. 
tars. Adornos erste Ästhetik-Vorlesung vom Wintersemester 1931/32 bedient sich eines bereits vorliegenden Werks, des Systems der Ästhetik von Johannes Volkelt, um in dessen kritischer Kommentierung die Umrisse der eigenen Ästhetik zu entwerfen (vgl. Adorno 1992). ${ }^{19}$ Auch in einer relativ frühen musikwissenschaftlichen Publikation verwendet Adorno das Verfahren des Kommentars, um Kritik geltend $\mathrm{zu}$ machen. ${ }^{20}$ In der unter dem Titel »Exkurse zu einem Exkurs« (M5, GS 18, S. 108-113) 1932 in den Blättern der städtischen Bühnen Essen publizierten Erwiderung auf den Beitrag »Exkurs über das Thema >Moderne Musik«" des Kunst- und Theaterkritikers Hans Georg Fellmann verfasst Adorno einen kurzen Stellenkommentar, der »einzelnen Sätzen und Worten des >Exkurses` Exkurse hinzufüg[t], in der Hoffnung, sie durchsichtig zu machen « (M5, GS 18, S. 109). Adorno beginnt jeweils einen Absatz mit einem Zitat aus Fellmanns »Exkurs«, etwa der Bemerkung zu den »auch für die musikalische Entwicklung gültigen biologischen Gesetze[n]« (M5, GS 18, S. 111 = Fellmann 1931, S. 13), und erläutert die Problematik von Fellmanns Argumentation und Wortwahl sodann in »Anmerkungen« (M5, GS 18, S. 108). Die Worte werden in der Reihenfolge, in der sie auch in Fellmanns Text stehen, zitiert, und die ersten drei Anmerkungen bis zu Fellmanns Begriff des stragischen Künstlertums` (M5, GS 18, S. $110=$ Fellmann 1931, S. 12) kommentieren unmittelbar aufeinanderfolgende Formulierungen. Letzteres suggeriert, dass Adorno nach dem Prinzip eines fortlaufenden Kommentars in der Lage gewesen wäre, Fellmanns ganzen Text Wort für Wort

19 In der Vorbemerkung zur Edition von Adornos Aufzeichnungen zur Ästhetik-Vorlesung von 1931/32 weist der Herausgeber Rolf Tiedemann auf Adornos diesbezüglichen, an Benjamin orientierten »entschlossenen Alexandrinismus« hin, der als Verfahren eine »alte, bis zu Kant vorherrschende Tradition der akademischen Vorlesung « wiederaufnimmt (Adorno 1992, S. 3537, hier S. 36).

20 Dass Kommentar und Kritik, die Adorno zusammendenkt - und meist zusammen nennt -, sich ausschlössen, gälte für das Verhältnis zum kanonisierten Text nur, wenn dieser tatsächlich als heiliger Text bestimmt würde; die ssäkularisierte Heiligkeitı entspricht bei Adorno aber nicht einer Relation, die keine Kritik erlauben würde, sondern einem Verhältnis zum Text, das diesen wie die alexandrinische Textkritik - die eine Kritik der Überlieferung ist, eine recensio beim Wort nimmt. Deshalb ist der Übergang vom Kommentar zur Kritik bzw. zum historischkritischen Kommentar, den Vismann (2006, S. 351f.) mit Foucault auf die Zeit nach der Renaissance ansetzt, dem Kommentar wenn auch nicht in rechtshistorischer, so doch in philologiehistorischer Perspektive inhärent. Der Philologe Nietzsche spricht denn auch, wie oben zitiert, mit völliger Selbstverständlichkeit von »kritische[n] Kommentaren« als ıspezifisch philologischer Aufgabe (KGW I/5, S. 55; vgl. zum philologischen Begriff der Kritik bei Nietzsche auch die Vorlesungsaufzeichnungen zur Encyclopädie der klassischen Philologie, KGW II/3, S. 373376). 
kritisch zu annotieren, ein umfassender Kommentar mithin eine umfassende Kritik bedeutet hätte.

Ist der Gegenstand dieser frühen Anmerkungen kein sheiliger Textı, sondern eine aus Adornos Sicht ebenso undurchdachte wie unreflektiert formulierte musikhistorische Publikation, die gleichwohl in ihrem Wortlaut zitiert und kritisiert wird, so nimmt Adorno in den Noten zur Literatur einen anderen Gegenstand, die Literatur, auf andere Weise beim Wort. Den Anmerkungen »Zur Schlußszene des Faust « schickt Adorno eine Bemerkung voraus, die im Kontext der Literatur sich für jenen Alexandrinismus ausspricht, den er als »Charakteristik Walter Benjamins« hervorgehoben hat. Adorno stellt das alexandrinische Verfahren, das »[p]rofane Texte wie heilige« anschaut, als »Antwort darauf, daß alle Transzendenz in die Profanität einwanderte« (NzL, GS 11, S. 129), in einen entschieden geschichtlichen Zusammenhang (siehe dazu auch Wussow 2014, S. 177-183), der ein nicht durch die Überlieferung vermitteltes Denken, das ohne Umweg einen Sinn aussagen will, mit Scham besetzt:

Für den Alexandrinismus, die auslegende Versenkung in überlieferte Schriften, spricht manches in der gegenwärtigen geschichtlichen Lage. Scham sträubt sich dagegen, metaphysische Intentionen unmittelbar auszudrücken; wagte man es, so wäre man dem jubelnden Mißverständnis preisgegeben. Auch objektiv ist heute wohl alles verwehrt, was irgend dem Daseienden Sinn zuschriebe, und noch dessen Verleugnung, der offizielle Nihilismus, verkam zur Positivität der Aussage, einem Stück Schein, das womöglich die Verzweiflung in der Welt als deren Wesensgehalt rechtfertigt, Auschwitz als Grenzsituation. Darum sucht der Gedanke Schutz bei Texten. (NzL, GS 11, S. 129)

Der Alexandrinismus ist für Adorno aber nicht nur durch die geschichtliche Situation bestimmt, sondern findet sich bereit im Gegenstand, der in den Noten zur Literatur kommentiert wird: in der Literatur selbst. Die Struktur einer Selbstkommentierung, wie sie Nietzsche zwischen Teilen seiner Werke und zwischen verschiedenen Werken postuliert, ist für Adorno nicht nur ein mögliches Verhalten zum Text unter anderen, sondern ein wesentliches Kennzeichen moderner Literatur, in der im Werk der »Autor als Kommentator « (ÄT, GS 7, S. 46) des Werks auftritt. Im Rundfunkvortrag zum »Standort des Erzählers im zeitgenössischen Roman« benennt Adorno die »Reflexion«, die »die reine Formimmanenz durchbricht« (NzL, GS 11, S. 45) als »Kommentar«, der in Prousts Recherche gewissermaßen mit dem Erzählen zusammenfällt und sich deshalb vom Erzählten kaum mehr unterscheiden lässt:

Wenn vollends bei Proust der Kommentar derart mit der Handlung verflochten ist, daß die Unterscheidung zwischen beiden schwindet, so greift damit der Erzähler einen Grundbestand im Verhältnis zum Leser an: die ästhetische Distanz. Diese war im traditionellen Roman unverrückbar. Jetzt variiert sie wie Kameraeinstellungen des Films: bald wird der 
Leser draußen gelassen, bald durch den Kommentar auf die Bühne, hinter die Kulissen, in den Maschinenraum geleitet. (NzL, GS 11, S. 46)

Nähert sich dieser Kommentarbegriff einem Begriff literarischer Reflexion an, durch die die Genese und die Struktur des Kunstwerks im Kunstwerk zur Darstellung gelangen, hat Adorno in den Noten zur Literatur durchaus auch einen Kommentar im engeren philologischen Sinn im Blick, der nicht nur als Verfahren, das allenfalls noch älteren, klassischen Werken entspräche, sondern gerade auch als Verhalten gegenüber den avanciertesten zeitgenössischen Texten seine Geltung erlangt. Im Vortrag zu den »Voraussetzungen« von Hans G. Helms' Sprachpartitur Fa:m' Ahniesgwow entspricht der »Philologie« des Kunstwerks, das einem "philologisch gelenkte[n] Assoziationszusammenhang" gehorcht (NzL, GS 11, S. 445), ein ebenso philologisch angelegter Kommentar: »Haben von jeher Dichtungen im Kommentar sich entfaltet, so ist diese auf den Kommentar angelegt wie jene deutschen Barockdramen, denen die gelehrten Schlesier ihre Scholien hinzufügten.« (NzL, GS 11, S. 445) Die Neigung zum Kommentar impliziert dabei auch, dass der literarische Text keineswegs auf ein Dargestelltes hin je schon festgeschrieben und gleichsam stillgestellt wäre, sondern sich im Kommentar überhaupt erst konstituiert. Der Kommentar entspricht einer Zeitstruktur, genauer: er erst entfaltet, so bemerkt Adorno in der Ästhetischen Theorie, die Kunstwerke, die »nicht zeitlos sich selbst gleich« sind (ÄT, GS 7, S. 507), und zwar sowohl im späteren Kommentar als auch je schon in der Literatur selbst. Bereits die Produktion des Kunstwerks hat an der philologischen Reflexion ihren Anteil, wie es im Essay zu Valéry heißt: »Indem der Produktionsprozeß zu dem der Reflexion auf das wird, was das sich entäußernde Werk von seinem Urheber ebenso wie vom Rezipierenden will, legitimiert sich das Denken über Kunst, dessen Fusion mit dem künstlerischen Prozeß bei Valéry das Normalbewußtsein permanent herausfordert. Das Werk entfaltet sich in Wort und Gedanken; Kommentar und Kritik sind ihm notwendig“ (NzL, GS 11, S. 188). Sind »Kommentar und Kritik« je schon im Kunstwerk angelegt und Bedingung seiner Entfaltung, so sieht Adorno in dieser Bewegung den Nukleus der Ästhetik, die das Telos des Kommentars ist. Aufgrund des »Rätselcharakter[s]«, den die Kunst »in sich selbst hat«, bedürfen die Kunstwerke

um ihrer eigenen Entfaltung, um ihres eigenen Lebens willen, eben des Kommentars und der Kritik. Und Kommentar und Kritik der Kunst sind zwar auf der einen Seite ein Element von ihrem eigenen Leben, sie sind aber andererseits nicht zu sistieren, sie treiben weiter, sind nicht aufzuhalten auf dem Weg eben zu einer Kunsttheorie, in der sie enden müssen. (NL 4/3, S. 34f.) 
Wenn insbesondere die moderne Literatur auf den Kommentar hin angelegt ist, so auch und gerade deshalb, weil sie prinzipielle Schwierigkeiten des Verstehens aufwirft. Die »Krisis der Verständlichkeit« (NzL, GS 11, S. 432), die prinzi-

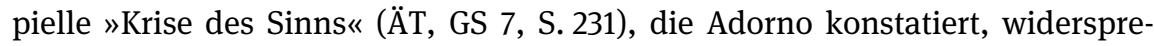
chen dem unvermittelten »Nachvollzug « eines geschlossenen »Sinnzusammenhangs« (NzL, GS 11, S. 431).

Demgegenüber zielt der Kommentar aufs Einzelne, aufs Detail. So wollen Adornos »Kleine Proust-Kommentare» in den Noten zur Literatur statt einer Gesamtschau der Recherche gerade durch Kommentare zu ausgewählten Passagen den Roman in den Blick bekommen:

Gegen kleine Kommentare zu einigen Abschnitten aus der >Suche nach der verlorenen Zeit ließe sich sagen, daß bei dem verwirrend reichen und krausen Gebilde der Leser mehr der orientierenden Überschau bedürfe, als daß er noch tiefer ins Einzelne verstrickt werden möchte, aus dem ohnehin nur schwer und mühsam der Weg zum Ganzen sich bahnen ließe. Der Einwand scheint mir der Sache nicht gerecht zu werden. An großen Übersichten fehlt es nicht länger. Das Verhältnis des Ganzen zum Detail jedoch bei Proust ist nicht das eines architektonischen Gesamtplans zu seiner Ausfüllung durchs Spezifische: eben dagegen, gegen das gewalttätig Unwahre einer subsumierenden, von oben her aufgestülpten Form hat Proust revoltiert. Wie die Gesinnung seines Werkes die herkömmlichen Vorstellungen von Allgemeinem und Besonderem herausfordert und ästhetisch ernst macht mit der Lehre aus Hegels Logik, das Besondere sei das Allgemeine und umgekehrt, beides sei durcheinander vermittelt, so kristallisiert sich das Ganze, allem abstrakten Umriß abhold, aus den ineinandergewachsenen Einzeldarstellungen. Eine jede birgt Konstellationen dessen in sich, was am Ende als Idee des Romans hervortritt. (NzL, GS 11, S. 203)

Adornos »Versenkung ins Bruchstück« (NzL, GS 11, S. 204) will dabei von den »Einzeldarstellungen« stets zur »Idee des Romans« (NzL, GS 11, S. 203) gelangen. Der Kommentar ist also durchaus nicht als Form zu verstehen, die sich im Detail vollends verlöre; vielmehr soll sich - »beharrend vorm Konkreten« (NzL, GS 11, S. 204) - im Besonderen das Allgemeine des Romans zeigen. Und ebenso wie im Vortrag zu Helms der Begriff des Kommentars eingesetzt wird, nachdem Adorno die Möglichkeit der Interpretation eingangs problematisiert hat (vgl. NzL, GS 11, S. 431f.), enden die »Kleinen Proust-Kommentare« mit einer Bemerkung, die die Geltung von Interpretationsversuchen einschränkt - dem Verfahren gemäß konkret auf eine bestimmte Stelle bezogen, einen Traum, in dem der bereits verstorbene Dichter Bergotte dem Erzähler erscheint, als wäre er noch am Leben (vgl. NzL, GS 11, S. 214). Die Stelle verweist auf den Satz aus der Recherche zurück, von dem Adorno im letzten der »Kommentare« ausgeht: »〉Der Gedanke, Bergotte sei nicht für alle Zeiten tot, ist demnach nicht völlig unglaubhaft.« (NzL, GS 11, S. 213; siehe dazu auch Wussow 2014, S. 180) Prousts 
"ganz und gar « metaphysischer Geist bewegt sich dabei, so Adorno, »inmitten einer Welt, welche die Sprache von Metaphysik verbietet« (NzL, GS 11, S. 213f.). Mithin stößt die Stelle mit dem im Traum wieder lebendigen Bergotte schon im Roman selbst, der gleichsam sein eigener kritischer Kommentar ist (vgl. NzL, GS 11, S. 46, und oben), an die Grenze des Denkbaren und ist deshalb auch ein Grenzfall der Interpretation, wie Adorno im letzten Satz der »ProustKommentare« vermerkt: »Jede Interpretation der Stelle bleibt hinter ihr zurück; nicht, wie das Cliché es will, weil ihrer künstlerische Würde höher stünde als der Gedanke, sondern weil sie selbst an der Grenze angesiedelt ist, auf die auch der Gedanke stößt.« (NzL, GS 11, S. 215)

\section{3}

Sind Adornos Kommentare anders als Nietzsches Selbstkommentierungen nicht auf das eigene Werk bezogen, ist der im zweiten Band der Noten zur Literatur nach den »Kleinen Proust-Kommentaren« folgende Text, »Wörter aus der Fremde«, gleichwohl eine Reflexion aufs eigene Schreiben - auf die Wortwahl in den »Proust-Kommentaren«, die Adorno gegenüber »Protestbriefe[n] wegen des angeblich übertriebenen Gebrauchs von Fremdwörtern nach der Radiosendung der Kleinen Proust-Kommentare« (NzL, GS 11, S. 216) rechtfertigt und dabei eine Reihe von Fremdwörtern, die er verwendet hat, einzeln annotiert (vgl. NzL, GS 11, S. 225-231). Wenn solche expliziten Reflexionen des Schreibens im publizierten Werk mitunter auch in programmatischer Weise hervortreten - etwa in der Frankfurter Antrittsvorlesung oder im Essay zum »Essay als Form « -, ist die Entstehung von Adornos Schriften, wie sie sich an den nachgelassenen Entwürfen nachvollziehen lässt, stets durch eine mikrologische Reflexion ihrer Form mitbestimmt. Ein Blick in die komplexen Entstehungszusammenhänge der Schriften verdeutlicht, dass Adorno nach eigenem Ermessen keineswegs immer schon druckreif formuliert, sondern Entwürfe vielfach überarbeitet hat (siehe Endres/Pichler/Zittel 2013 zur Genese der Ästhetischen Theorie). Wenn davon auszugehen ist, dass sowohl bei Adorno als auch bei Nietzsche das Schreiben nicht einfach die äußere Form des Denkens, sondern das Denken selbst betrifft - nicht nur Beiwerk des Gedankens ist, sondern die sprachliche Form einen »durch nichts ganz zu zerbrechenden Zusammenhang« (ND, GS 6, S. 66) mit dem Denken bildet -, verlangen die komplexen Textgenesen, die sich bei beiden Autoren finden, nach einer Analyse, die auch die Zusammenhänge und Interferenzen zwischen Gehalt und Prozess des Schreibens berücksichtigt. Soll dabei der Kommentar, den Nietzsche und Adorno als konstitutives Element 
ihres Werks begreifen, auch als Begriff zur Bestimmung von Aspekten des Schreibprozesses dienen, so erlaubt es die große Komplexität der Entwürfe keineswegs, diese damit auf einen einfachen Nenner zu bringen. Vielmehr kann das Kommentarverhältnis, wie es sowohl Nietzsche als auch Adorno reflektiert haben, auch als ein Verfahren im Schreiben begriffen werden, das neben anderen Schreibverfahren hervortritt, wo in Entwürfen einzelne Teile der Manuskripte $\mathrm{zu}$ anderen Teilen in einem kommentierenden Verhältnis stehen. ${ }^{21}$

Sind mit den Einfügungen und Ersetzungen in Nietzsches Manuskripten jeweils bestimmte Veränderungen intendiert, betreffen nicht alle Zusätze den Wortlaut eines entworfenen Textes; sie können eine gewisse Eigenständigkeit bewahren, die gleichwohl in engem Verhältnis zu den Entwürfen oder Notaten steht, auf die sie sich beziehen. Soweit sie bereits Geschriebenes erläutern oder verdeutlichen, aber nicht unmittelbar Teil davon sind, weisen diese Ergänzungen eine kommentierende Struktur auf. In der Topographie der Entwürfe lässt sich mithin differenzieren zwischen Einfügungen, die einen bereits entworfenen Wortlaut zu verändern beabsichtigen, und Ergänzungen, die diesen unberührt lassen, aber mit ihm eng zusammenhängen. Das hat zur Folge, dass eine Textkonstituierung, die einen linearen Text herstellt, nur über Umwege die kommentierenden Ergänzungen in diesen Text integrieren kann.

Dies soll nun an einem vergleichsweise übersichtlichen Beispiel erläutert werden: den nachgelassenen Notaten »Zur Vorrede der >Morgenröthe« « (KGW IX/5, W I 8, S. 65f.). Die Bemerkungen »Zur Vorrede der > Morgenröthe« « gehören in den Kontext der Vorreden, die Nietzsche zu den Neuauflagen bereits publizierter Bücher ebenso wie zu geplanten neuen Veröffentlichungen im Arbeitsheft W I 8 entwirft. Die in schwarzer Tinte zunächst notierte Grundschicht weist nur wenige unmittelbare Korrekturen auf. Sowohl auf S. 65 des Arbeitsheftes als auch auf der gegenüberliegenden Seite finden sich jedoch spätere »Einfügungen und Zusätze «22, die sich z. T. direkt in die bereits niedergeschriebenen Formulierungen eingliedern lassen. Gleich die erste Zeile unter dem Titel auf S. 65, die ihrerseits erst nach den folgenden Zeilen eingefügt worden ist, weist mehrschichtige Korrekturen auf, die ohne größere Schwierigkeiten die beabsichtige neue Formulierung erkennen lassen. Oben auf der Seite steht zunächst: »Ver-

21 Ein weiterer Typus des Kommentars, der sich sowohl bei Nietzsche als auch bei Adorno findet, sind die Annotationen in und zu Büchern, die bei beiden Autoren eng mit der Genese eigener Texte zusammenhängen.

22 So die Terminologie in den Erläuterungen zu den textkritischen Auszeichnungen (KGW IX/5, S. IX). Ich danke Beat Röllin (Basel) für luzide Hinweise zur Textgenese der Notate »Zur Vorrede der `Morgenröthe« $«$. 
such über Moral zu denken, ohne unter dem Zauber ihrer Verehrung zu stehen. -« Diese Formulierung wird durch Einfügungen verändert zu: »Versuch über Moral zu denken, ohne unter ihrem Zauber zu stehen, mißtrauisch gegen die Überlistung ihrer schönen Gebärden u. Blicke." (KGW IX/5, W I 8, S. 65; siehe auch Abb. 1) Während sich auch in der unteren Seitenhälfte Veränderungen des Wortlauts erkennen lassen (die Zeilen 28, 30, 40 und 44 betreffend), finden sich als Marginalien neben Nietzsches Zusammenfassung des zuvor erläuterten »Kantsche[n] Kriticismus« und des »Hegelische[n] Ausweg[s]«, die nach dem Stichwort »Kurz« unter den Ziffern »1« und »2« notiert sind, zwei Formulierungen, die sich nicht mit den danebenstehenden Bemerkungen $\mathrm{zu}$ neuen Formulierungen zusammenfassen lassen. Zum ersten, kantischen Punkt (»Gott ist uns unerkennbar u. unnachweisbar «) notiert Nietzsche in den linken Randgang: »Hintersinn der / erkennt.theor. Be=/ wegung «, zum zweiten, hegelischen Punkt (in der Grundschicht: »Gott ist etwas Werdendes, und wir gehören dazu, eben mit unseren Idealen«) ebenda: »Hintersinn der hi=/ storisirenden Bewegung«. Dass sich die zwei Bemerkungen aufgrund von Tintenfarbe und Schriftduktus nicht von den gleich danebenstehenden Umformulierungen $\mathrm{zu}$ `Gott als etwas Werdendem` unterscheiden lassen, könnte dazu verleiten, sie ebenfalls als direkte Ergänzungen zum bereits Niedergeschriebenen zu werten. In der Textkonstitution der Kritischen Gesamtausgabe greift der Herausgeber deshalb zu dem Mittel, die beiden kurzen Bemerkungen mithilfe der editorischen Hinzufügung von Gedankenstrichen in den Text einzuordnen:

1) Gott ist uns unerkennbar und unnachweisbar - Hintersinn der erkenntnißtheoretischen Bewegung

2) Gott ist nachweisbar, aber als etwas Werdendes -, und wir gehören dazu, eben mit unsrem Drang zum Idealen - Hintersinn der historisirenden Bewegung (NL 1885/1886, 2[165], KGW VIII/1, S. 146; Herv. FCh)

Diese editorische Lösung ergibt einen nachvollziehbaren Textverlauf, verstellt aber bis zu einem gewissen Grad den Blick auf die Logik der Notation. Denn die zwei konjizierten Gedankenstriche, die im Manuskript nicht stehen, sind lediglich Statthalter eines kommentierenden Verhältnisses, das durch die marginale Notation Nietzsches, wie sie sich in Faksimile und Transkription erkennen lässt (siehe Abb. 1 und 2), deutlicher als in der Textkonstitution hervortritt. Die zwei Marginalien sind nicht Teil eines fortlaufenden Textes, sondern explizieren die theologisch zugespitzte Zusammenfassung der kantschen und hegelschen Philosophie als Nietzsches Kritik der Epistemologie und des Historismus. Handelt es sich bei der Doppelseite um Notate zu einer Vorrede, so haben Vorreden in Nietzsches brieflicher Sicht eine kommentierende Funktion im Verhältnis zum 
Werk (vgl. KGB III/5, Bf. 908, und oben). Die in diesem Sinn auf einen Kommentar zu einem bereits publizierten Werk angelegte Doppelseite weist mit den zwei Marginalien ihrerseits kleine kommentierende Elemente auf, die die Genese der Vorrede mitbestimmen. Anders als die expliziten Kommentare zum eigenen Schreiben, die sich ebenfalls in den Arbeitsheften finden - wie dem bekannten Notat »Ich achte die Leser nicht mehr: wie könnte ich für Leser schreiben? .. Aber ich notiere mich, für mich « (KGW IX/6, W II 1, S. 1; siehe dazu Pichler 2014, S. 69) -, sind diese kleinen Kommentare nicht aufs Schreiben insgesamt, sondern auf bestimmte andere Notate bezogen. Sie zeigen aber gerade deshalb eine Logik des Schreibens an, die auch in ihren Mikrostrukturen kommentierende Relationen zeitigt.

Demgegenüber lässt sich in Adornos Schreibsystem - zu dem Manu- und Typoskripte, insbesondere das Diktat und die handschriftliche Überarbeitung des diktierten Typoskripts mit anschließender maschinenschriftlicher Abschrift und allenfalls erneutem Korrekturdurchgang gehören- sowohl bei kleineren Arbeiten (vgl. etwa die 2. Fassung des Helms-Essays, TS 38863-38887) als auch und insbesondere in den Entwürfen zur Ästhetischen Theorie ein Typus von kommentierender Bemerkung verzeichnen, der auf spezifische Weise die jeweilige weitere Ausarbeitung des Entwurfs betrifft. In den »Prolegomena zu einer Textkritischen Edition der Ästhetischen Theorie» sprechen die Herausgeber in diesem Zusammenhang von »Metakommentare[n]« (Endres/Pichler/Zittel 2013, S. 186), die sich sowohl in den gesondert überlieferten sogenannten >Regiebemerkungen ‘ Adornos (siehe dazu Endres/Pichler/Zittel 2013, S. 178) als auch in den Typoskripten zur Ästhetischen Theorie finden. In den mehrschichtigen Typoskripten betrifft dies nicht Adornos Eingriffe in den entworfenen Text mit blauer Tinte, sondern die von den direkten Eingriffen $\mathrm{zu}$ differenzierenden Aufzeichnungen mit Bleistift, »bei denen es sich um kleinere Notate, Anweisungen an Elfriede Olbrich, Regieanweisungen, offene Fragen zur Textgestaltung und die Kommunikation mit Gretel Adorno handelt« (Endres/Pichler/Zittel 2013, S. 191).

So finden sich etwa auf den Typoskriptblättern, die die Grundlage für die Edition der Abschnitte $\mathrm{zu}$ »Intention und Sinn « und zur »Krise des Sinns « in der Suhrkamp-Ausgabe bilden (ÄT, GS 7, S. 226-235), eine ganze Anzahl von Bleistiftanmerkungen, die jeweils zum einen erläutern, was allenfalls noch an der Textgestaltung konkret zu ändern ist, wenn es etwa am oberen Rand der Seite 
heißt: »hier eventuell neuer Absatz« (TS 18009). ${ }^{23}$ Zum anderen sind diese in Blei notierten Bemerkungen Erläuterungen wesentlicher Elemente des Gedankengangs - sie kommentieren, wovon auf einer Seite die Rede ist oder in der weiteren Ausarbeitung die Rede sein soll. Dabei verschränken die Aufzeichnungen die Kommentierung des Gedankengangs und die Kommentierung seiner Darstellung. Das zeigt sich gerade auch in der materiellen Zusammenführung beider nur scheinbar getrennten Aufzeichnungstypen: Die Bemerkung »hier eventuell neuer Absatz« folgt unmittelbar unter Bleistiftbemerkungen zur Sinnkrise: »Krisis des Sinnes 2) Steigende Unmöglichkeit daß Werke sich als Sinnzusammen-/hang konstituieren / Selbstkritik der Werke = Kritik am Sinn" (TS 18009). Das Notat erläutert den darunterstehenden komplexen Entwurf und setzt mit der Gleichung "Selbstkritik der Werke = Kritik am Sinn« die Kommentarstruktur moderner Literatur, wie Adorno sie in den Noten zur Literatur umreißt und auch in der Ästhetischen Theorie benennt - Kommentar und Kritik beginnen je schon im modernen Kunstwerk selbst - mit der "Krisis des Sinnes« in eins. Das Notat bezieht sich auf eine Formulierung in der Mitte der Seite: »Kritische Selbstreflexion, wie sie jeglichem Kunstwerk inhätiert [sic], schärft dessen Empfindlichkeit gegen alle die Momente in ihm, die herkömmlich Sinn bekräftigen « (TS 18009; vgl. ÄT, GS 7, S. 229). Die Wendung »Kritische Selbstreflexion" ersetzt dabei handschriftlich die maschinenschriftliche Formulierung "Die objektive Selbstkritik«, die der Kommentar am oberen Rand wörtlich aufgreift und in eine Gleichung verwandelt. Es handelt sich bei diesem Notat also weder um einen direkten Eingriff in den maschinenschriftlichen Text noch um die Aufzeichnung eines vom Text unabhängigen Gedankens; vielmehr ist es eine >kritische Selbstreflexion` der entworfenen ästhetischen Theorie, die sich allerdings von der $"[\mathrm{k}]$ ritischen Selbstreflexion, wie sie jeglichem Kunstwerk inhä[r]iert«, durchs »Medium des Begriffs« (ÄT, GS 7, S. 531) unterscheidet. ${ }^{24}$ Adornos kommentierende Bleistiftbemerkungen betreffen eine Ästhetik, die in den Entwürfen zur Ästhetischen Theorie keine feste Gestalt hat, sondern deren Kritik ihrer Darstellungsverfahren sich durch die Struktur der Entwürfe abbildet.

23 Diese und die folgenden Transkriptionen stammen von den Herausgebern der in Vorbereitung befindlichen Textkritischen Edition der Ästhetischen Theorie (siehe Endres/Pichler/Zittel 2013).

24 Zum »aporetischen Nexus von Kunst und Philosophie« bei Adorno siehe Geulen 2002, S. $120 \mathrm{zu}$ Adornos Unterscheidung von interpretativer und ästhetischer (dem Kunstwerk eigener) Rede Menke 1988, S. $122 f$. 
Die kommentierenden Strukturen, die sich in Entwürfen von Nietzsche und Adorno vermerken lassen, sind dabei nicht vollkommen kongruent mit Begriff und Textverfahren des Kommentars. Weder sind sie wie bei Nietzsche eine vom Autor explizit erörterte Setzung, die die Lektüre anleiten will, noch entwerfen sie stets das von Adorno geforderte kritische Verhältnis zur philosophischen und literarischen Tradition. Gleichwohl entsprechen sie auf mikrologischer Ebene dem kommentierenden Selbstverhältnis, das Nietzsche entwirft, und der von Adorno umrissenen Charakteristik des Kommentars, in dem sich ein seine Sprachlichkeit reflektierendes Denken entfaltet. Der Kommentar korrespondiert sowohl bei Nietzsche als auch bei Adorno mit einer philologisch geprägten Form, die nicht nur als technisches Verfahren, sondern als Denkform begriffen wird. 


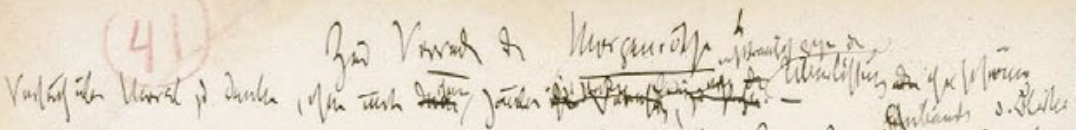

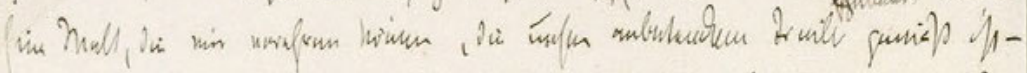

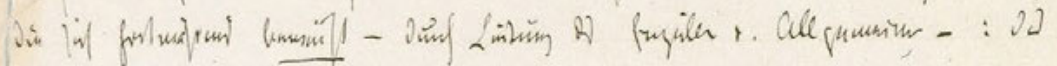

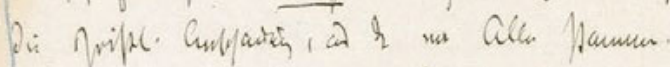

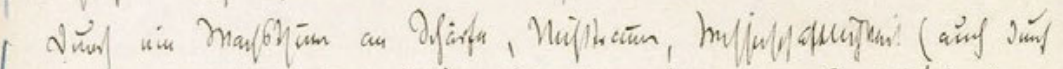

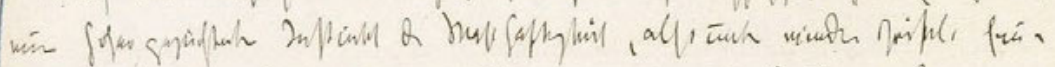

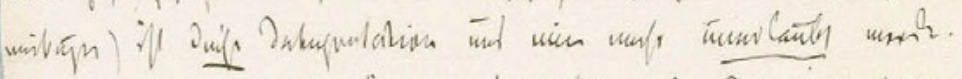

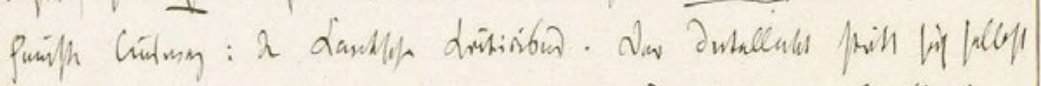

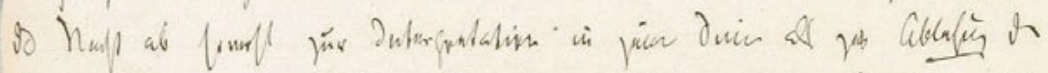

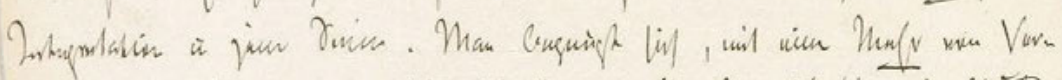

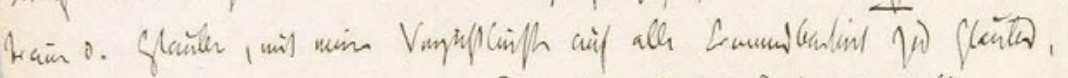

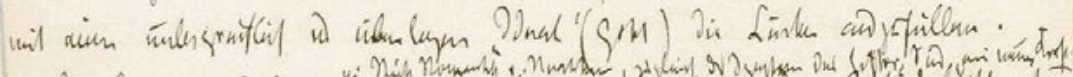

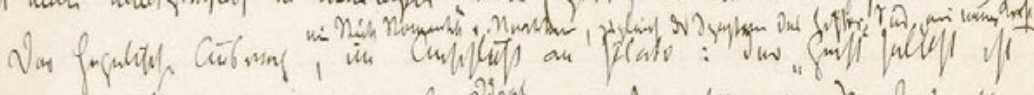

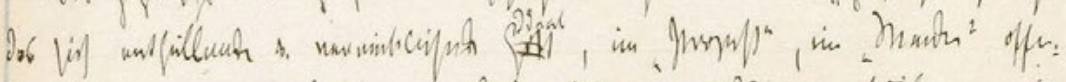

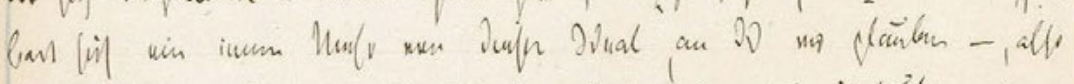

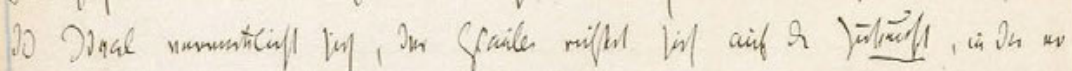

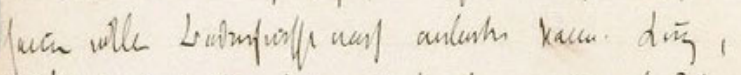

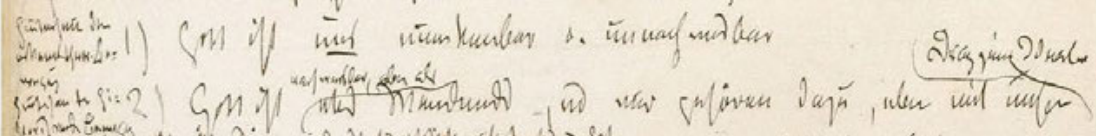

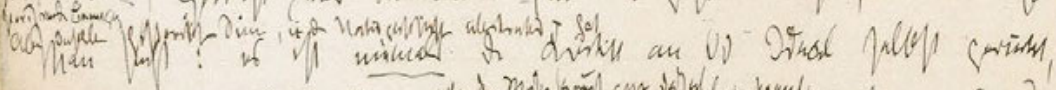

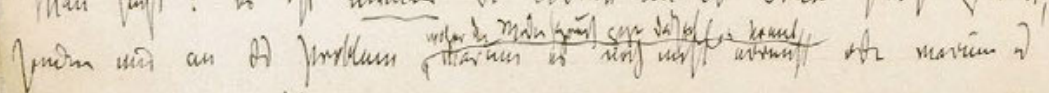

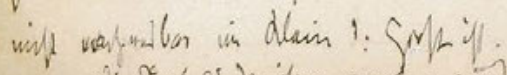

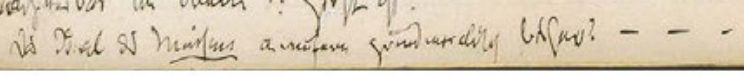

Abb. 1: W I 8, S. 65 (Quelle: Klassik Stiftung Weimar) 
$W 18$

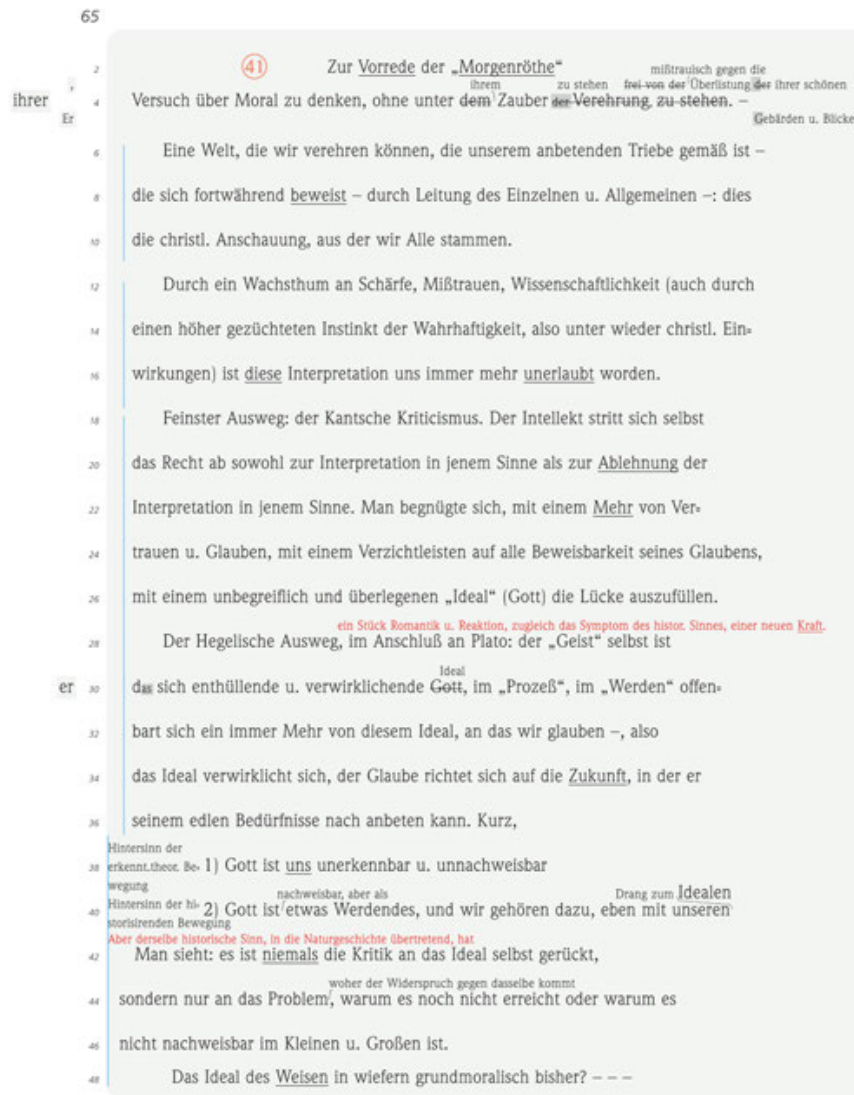

Abb. 2: Transkription W I 8, S. 65 (KGW IX/5) 


\section{Literaturverzeichnis}

Abel, Günter (1998): Nietzsche. Die Dynamik der Willen zur Macht und die ewige Wiederkehr. 2. Aufl. Berlin, New York: De Gruyter.

Adelung, Johann Christoph (1793-1801): Grammatisch-kritisches Wörterbuch der hochdeutschen Mundart, mit beständiger Vergleichung der übrigen Mundarten, besonders aber der oberdeutschen. 2. Aufl. Leipzig.

Adorno, Theodor W. (1992): „Aufzeichnungen zur Ästhetik-Vorlesung von 1931/32«. In: Frankfurter Adorno-Blätter 1, S. 35-90.

Assmann, Aleida (1995): „Der Eigen-Kommentar als Mittel literarischer Traditionsstiftung. Zu Edmund Spensers The Shepheardes Calender«. In: Jan Assmann/Burkhard Gladigow (Hrsg.): Text und Kommentar. Archäologie der literarischen Kommunikation IV. München: Fink, S. 355-373.

Assmann, Jan (1995): „Text und Kommentar. Einführung«. In: Jan Assmann/Burkhard Gladigow (Hrsg.): Text und Kommentar. Archäologie der literarischen Kommunikation IV. München: Fink, S. 9-33.

Auerochs, Bernd (2014): „Text und Kommentar bei Walter Benjamin«. In: Nicolas Berg/Dieter Burdorf (Hrsg.): Textgelehrte. Literaturwissenschaft und literarisches Wissen im Umkreis der Kritischen Theorie. Göttingen: Vandenhoeck \& Ruprecht, S. 125-137.

Aust, Hugo (1993): »Dichter-Kommentar. Am Beispiel der Fußnoten- und Anmerkungspraxis im historischen Roman«. In: Beihefte zu editio 5, S. 93-98.

Benne, Christian (2005): Nietzsche und die historisch-kritische Philologie. Berlin, New York: De Gruyter.

Endres, Martin/Pichler, Axel/Zittel, Claus (2013): »'Noch offen`. Prolegomena zu einer Textkritischen Edition der Ästhetischen Theorie Adornos«. In: editio 27, S. 173-204.

Fellmann, Hans Georg (1931): „Exkurs über das Thema `Moderne Musik««. In: Der Scheinwerfer 6, S. 12-14.

Fietz, Rudolf (1992): Medienphilosophie. Musik, Sprache und Schrift bei Friedrich Nietzsche. Würzburg: Königshausen \& Neumann.

Figal, Günter (2000): »Nietzsches Philosophie der Interpretation«. In: Nietzsche-Studien 29, S. 1-11.

Figl, Johann (1982): Interpretation als philosophisches Prinzip. Friedrich Nietzsches universale Theorie der Auslegung im späten Nachlaß. Berlin, New York: De Gruyter.

Foucault, Michel (1991): Die Ordnung des Diskurses. Übers. v. Walter Seitter. Frankfurt am Main: Fischer.

Fuhrmann, Manfred (1985): „Kommentierte Klassiker? Über die Erklärungsbedürftigkeit der klassischen deutschen Literaturu. In: Gottfried Honnefelder (Hrsg.): Warum Klassiker? Ein Almanach zur Eröffnungsedition der Bibliothek deutscher Klassiker. 2. Aufl. Frankfurt am Main: Deutscher Klassiker Verlag, S. 37-57.

Geulen, Eva (2002): Das Ende der Kunst. Lesarten eines Gerüchts nach Hegel. Frankfurt am Main: Suhrkamp.

Geulen, Eva (2015): »Selbst-Kommentar«. In: Trajekte 31, S. 1-3.

Goethe, Johann Wolfgang (1994): West-östlicher Divan. Hrsg. v. Hendrik Birus. In: J. W. Goethe: Sämtliche Werke, Briefe, Tagebücher und Gespräche. Bd. I/3. Frankfurt am Main: Deutscher Klassiker Verlag. 
Groddeck, Wolfram (1983): »)Ein andres Wort für Musikı. Zu Friedrich Nietzsches VenedigGedicht«. In: Harald Hartung (Hrsg.): Gedichte und Interpretationen. Bd. 5.: Vom Naturalismus bis zur Jahrhundertmitte. Stuttgart: Reclam, S. 20-32.

Groddeck, Wolfram (1991): Die »Dionysos-Dithyramben«. Bedeutung und Entstehung von Nietzsches letztem Werk. Berlin, New York: De Gruyter.

Gumbrecht, Hans Ulrich (2003): Die Macht der Philologie. Über einen verborgenen Impuls im wissenschaftlichen Umgang mit Texten. Übers. v. Joachim Schulte. Frankfurt am Main: Suhrkamp.

Hegel, Georg Wilhelm Friedrich (2016): Gesammelte Werke. In Verbindung mit der Deutschen Forschungsgemeinschaft hrsg. v. der Nordrhein-Westfälischen Akademie der Wissenschaften und der Künste. Bd. 30,1: Vorlesungen über die Geschichte der Philosophie. Hrsg. v. Klaus Grotsch. Hamburg: Meiner.

Kästle, David/Jansen, Nils (2014) (Hrsg.): Kommentare in Recht und Religion. Tübingen: Mohr Siebeck.

Menke, Christoph (1988): Die Souveränität der Kunst. Ästhetische Erfahrung nach Adorno und Derrida. Frankfurt am Main: Athenäum.

Pfeiffer, Rudolf (1968): History of Classical Scholarship from the Beginnings to the End of the Hellenistic Age. Oxford: Clarendon Press.

Pichler, Axel (2014): Philosophie als Text - Zur Darstellungsform der Götzen-Dämmerung. Berlin, Boston: De Gruyter.

Plass, Ulrich (2007): Language and History in Theodor W. Adorno's Notes to Literature. New York, London: Routledge.

Primavesi, Patrick (1998): Kommentar, Übersetzung, Theater in Walter Benjamins frühen Schriften. Frankfurt am Main, Basel: Stroemfeld.

Sanchiño Martínez, Roberto (2013): „Aufzeichnungen eines Vielfachen«. Zu Friedrich Nietzsches Poetologie des Selbst. Bielefeld: transcript.

Schironi, Francesca (2012): „Greek Commentaries«. In: Dead Sea Discoveries 19, S. 399-441.

Scholze, Britta (2000): Kunst als Kritik. Adornos Weg aus der Dialektik. Würzburg: Königshausen \& Neumann.

Schrift, Alan D. (1990): Nietzsche and the Question of Interpretation: Between Hermeneutics and Deconstruction. New York, London: Routledge.

Seneca, Lucius Annaeus (1987): Philosophische Schriften. Bd. 4: An Lucilius. Briefe über Ethik 70-124, [125]. Lateinisch und deutsch. Hrsg. und übers. v. Manfred Rosenbach. 2. Aufl. Darmstadt: Wissenschaftliche Buchgesellschaft.

Sommer, Andreas Urs (2013a): '»Glossarium<, ’Commentar` oder `Dynamit‘? Zu Charakter, Konzeption und Kontext von Jenseits von Gut und Böse«. In: Marcus Andreas Born/Axel Pichler (Hrsg.): Texturen des Denkens. Nietzsches Inszenierung der Philosophie in Jenseits von Gut und Böse. Berlin, Boston: De Gruyter, S. 69-86.

Sommer, Andreas Urs (2013b): Historischer und kritischer Kommentar zu Friedrich Nietzsches Werken. Hrsg. v. der Heidelberger Akademie der Wissenschaften. Bd. 6/2: Kommentar zu Nietzsches Der Antichrist, Ecce homo, Dionysos-Dithyramben, Nietzsche contra Wagner. Berlin, Boston: De Gruyter.

Stegmaier, Werner (2012): Nietzsches Befreiung der Philosophie. Kontextuelle Interpretation des V. Buchs der Fröhlichen Wissenschaft. Berlin, Boston: De Gruyter.

Stingelin, Martin (2002): ")er war im Grunde der eigentliche Schriftsteller, während ich bloss der Autor warı. Friedrich Nietzsches Poetologie der Autorschaft als Paradigma des französischen Poststrukturalismus (Roland Barthes, Gilles Deleuze, Michel Foucault)«. In: Hein- 
rich Detering (Hrsg.): Autorschaft. Positionen und Revisionen. Stuttgart, Weimar: Metzler, S. 80-106.

Vismann, Cornelia (2006): »Benjamin als Kommentator«. In: Eva Horn/Bettine Men-

ke/Christoph Menke (Hrsg.): Literatur als Philosophie - Philosophie als Literatur. München: Fink, S. 347-362.

Westerdale, Joel (2013): Nietzsche's Aphoristic Challenge. Berlin, Boston: De Gruyter.

Wilcox, John T. (1997): "What Aphorism Does Nietzsche Explicate in Genalogy of Morals, Essay III? « In: Journal of the History of Philosophie 35:4, S. 593-610.

Wilcox, John T. (1998): "That Exegesis of an Aphorism in Genealogy III: Reflections on the Scholarship«. In: Nietzsche-Studien 27, S. 448-462.

Wussow, Philipp von (2014): „Adorno über literarische Erkenntnis«. In: Nicolas Berg/Dieter Burdorf (Hrsg.): Textgelehrte. Literaturwissenschaft und literarisches Wissen im Umkreis der Kritischen Theorie. Göttingen: Vandenhoeck \& Ruprecht, S. 159-183. 
Bereitgestellt von | UZH Hauptbibliothek / Zentralbibliothek Zürich Angemeldet Heruntergeladen am | 06.04.18 10:55 